\title{
Lysosomal Function Induction
}

National Cancer Institute

\section{Source}

National Cancer Institute. Lysosomal Function Induction. NCI Thesaurus. Code C40786.

Lysosomal Function Induction involves initiation of activities of diverse glycoprotein hydrolytic enzymes, active at acid pH, with structure-linked latency, and stored in single unit membrane-bound and morphologically heterogeneous cytoplasmic vesicles. 